\title{
ORIGINAL RESEARCH \\ MR Imaging of Familial Creutzfeldt-Jakob Disease: A Blinded and Controlled Study
}

\section{R.K. Fulbright \\ C. Hoffmann \\ H. Lee}

A. Pozamantir

J. Chapman

I. Prohovnik
BACKGROUND AND PURPOSE: The E200K mutation of the PRNP (prion protein) gene is the most common cause of familial Creutzfeldt-Jakob disease (fCJD), which has imaging and clinical features that are similar to the sporadic form. The purpose of this study was to conduct a controlled and blinded evaluation of the sensitivity and specificity of MR imaging in this unique population.

MATERIALS AND METHODS: We compared the MR imaging characteristics of 15 early stage familia CJD patients (age, $60 \pm 7$ years) with a group of 22 healthy subjects from the same families (age, $61 \pm$ 8 years). MR imaging included diffusion-weighted imaging (DWI), T2-weighted fast spin-echo imaging, and a fluid-attenuated inversion recovery (FLAIR) sequence. The scans were rated for abnormalities by an experienced neuroradiologist blind to diagnosis, group assignment, age, and sex.

RESULTS: Thirteen of $15 \mathrm{fCJD}$ subjects had abnormal MR imaging. FLAIR signal intensity abnormality in the caudate or putamen nuclei demonstrated a sensitivity of $87 \%$ and specificity of $91 \%$. DWI abnormality in the caudate nucleus showed a sensitivity of $73 \%$ and a specificity of $100 \%$. Abnormalities in the thalamus (6 patients), cingulate gyrus (6 patients), frontal lobes (4 patients), and occipital lobes (3 patients) were best detected with DWI. No signal intensity abnormalities were demonstrated in the cerebellum. T2-weighted and T1-weighted sequences were uninformative.

CONCLUSIONS: FLAIR and DWI abnormalities in the caudate nucleus and putamen offer the best sensitivity and specificity for diagnosing fCJD. Our findings support recent recommendations that MR imaging should be added to the diagnostic evaluation of CJD.
$\mathbf{C}^{\mathrm{r}}$ eutzfeldt-Jakob disease (CJD) is the most common human prion disease. It is a rare neurodegenerative disorder that is progressive and invariably fatal, with nearly $90 \%$ of patients dying within 1 year of diagnosis. ${ }^{1}$ CJD occurs in approximately 1 person per 1 million people per year worldwide. ${ }^{1}$ The most common form is sporadic CJD (sCJD), which occurs randomly without a known risk factor and accounts for $85 \%-90 \%$ of cases. Familial or hereditary CJD (fCJD), seen in $5 \%-10 \%$ of cases, is caused by mutations in the gene that controls formation of the normal prion protein on chromosome 20. ${ }^{1}$ The most common pathogenic mutation is the E200K mutation. The risk of fCJD is transmitted in an autosomal dominant inheritance pattern, with nearly $100 \%$ penetrance. $^{2}$

The imaging findings in SCJD typically consist of cortical atrophy and hyperintensities in the basal ganglia, thalamus, and cortex on fluid-attenuated inversion recovery (FLAIR) and diffusion-weighted imaging (DWI).$^{3-13}$ fCJD has imaging findings and neuropathology that are, in general, similar to the most common forms of sCJD; however, most imaging studies on fCJD consist primarily of case reports or studies focused on sCJD that combine a few fCJD patients into a single CJD patient sample. ${ }^{8,14-22}$ fCJD studies are limited not only by small sample sizes but also by nonstandardized imaging protocols

Received April 2, 2008; accepted after revision May 27.

From the Department of Radiology (R.K.F., I.P.), Yale University School of Medicine, New Haven, Conn; Departments of Radiology (C.H.) and Neurology (J.C.), Chaim Sheba Medical Center, Ramat Gan, Israel; Departments of Psychiatry (H.L., A.P., I.P.) and Radiology (I.P.), Mount Sinai School of Medicine, New York, NY; and Sackler Faculty of Medicine (J.C.), Tel Aviv University, Tel Aviv, Israel.

This work was supported by National Institutes of Health grant R01 NS43488.

Please address correspondence to Robert K. Fulbright, Yale University School of Medicine, MRRC, The Anlyan Center N137, 300 Cedar St, P0 Box 208043, New Haven, CT 065208043; E-mail: robert.fulbright@yale.edu

indicates article with supplemental on-line table.

DOI 10.3174/ajnr.A1217 and clinical and pathophysiologic heterogeneity. The complex interactions with disease duration and cognitive and neurologic severity have also made it difficult to interpret studies, especially because the sample sizes are small. ${ }^{23}$

To address the difficulties of clinical research in this area, we initiated a study of fCJD occurring among Libyan Jews living in Israel that is caused by familial transmission of the E200K mutation. ${ }^{24-26}$ In a preliminary report, it was demonstrated that 4 patients with fCJD due to the E200K mutation had gray matter atrophy and decreased apparent diffusion coefficient (ADC) in the basal ganglia. Signal intensity hyperintensities were seen in the basal ganglia and thalamus with FLAIR and DWI. ${ }^{27}$ The sample size in that study was insufficient to calculate sensitivity and specificity. Here we describe a rigorously blinded and controlled evaluation of $\mathrm{MR}$ imaging findings in a larger number of fCJD patients.

\section{Methods}

\section{Patients and Control Subjects}

This analysis includes the first MR studies obtained in 39 consecutive subjects who met the inclusion criteria (below). There were 17 subjects with the E200K mutation who were diagnosed clinically with fCJD and 22 healthy control subjects from the same families.

The inclusion and exclusion criteria for the subjects (here denoted group S, patients) included a clinical diagnosis of CJD and the presence of the E200K mutation. The clinical diagnosis of CJD is an evolving field, and newer CSF markers and MR imaging criteria will probably by added soon. ${ }^{28}$ Our criteria are consistent with the conventional World Health Organization definitions and required that the subjects have rapidly progressive neurologic dysfunction and behavioral changes of less than 2 years of duration with at least 2 of the following features being present at some point during their illness: dementia, myoclonus, visual or cerebellar symptoms, pyramidal or extrapyramidal dysfunction, or akinetic mutism. The relevant E200K 
mutation (prion protein gene, PRNP 20pter-p12) characteristic of fCJD in this ethnic group ${ }^{24,29}$ was determined for all of the subjects by methods described previously. ${ }^{2}$ Two of the $17 \mathrm{fCJD}$ patients were excluded from the current analysis because their first MR imaging was obtained in the process of conversion from healthy to symptomatic, and it was unclear whether they met clinical criteria for a diagnosis of CJD at that point in time. One of these 2 subjects converted to a full CJD pattern 3 months later, and the second subject died before a follow-up MR image could be obtained. In the remaining 15 subjects composing the patient group, 14 were followed to death to confirm the diagnosis, and 1 subject remains alive. The inclusion and exclusion criteria for the controls were as follows: age greater than or equal to 50 years to have age-matched groups, healthy by history, medical and neurologic examination, and neuropsychologic testing, belong to a family with known members who carried the E200K mutation, and no significant history of drug or alcohol abuse.

Neurologic examination included the Mini-Mental State Examination (MMS) ${ }^{30}$ and a structured semiquantitative checklist of symptom severity by the CJD Rating Scale (CRS), which was designed specifically to quantify CJD symptoms. ${ }^{31}$ The CRS screens for the presence of positive neurologic signs and symptoms of the visual and oculomotor, brain stem, cerebellar, pyramidal, extrapyramidal, and spinal and peripheral systems.

\section{Imaging}

MR images were performed on a 1.5T Signa Excite MR system (GE Healthcare, Waukesha, Wis) by using a standard quadrature head coil. Sequences included a rapid scout, followed by DWI using a single-shot, echo-planar, spin-echo sequence acquired in the axial-oblique plane with parameters of TR $5000 \mathrm{~ms}$, TE $85 \mathrm{~ms}$, FOV of $24 \mathrm{~cm}$, and imaging matrix of $128 \times 128$, with b-values of $0 \mathrm{~s} / \mathrm{mm}^{2}$ (T2weighted image) and $1000 \mathrm{~s} / \mathrm{mm}^{2}$ along 3 orthogonal directions, and 3 -mm contiguous sections resulting in 48 sections. The average DWI image was calculated as the mean of the individual signal intensities in each of the 3 orthogonal diffusion vector directions. An ADC map (in units of $\mu \mathrm{m}^{2} / \mathrm{s}$ ) was constructed automatically from the average DWI image and the T2-weighted image $(b=0)$ by standard formulas. ${ }^{16} \mathrm{~A}$ FLAIR sequence had parameters of TR $9000 \mathrm{~ms}$, TE $120 \mathrm{~ms}$, and TI $2200 \mathrm{~ms}$, and a T2-weighted fast spin-echo (FSE) sequence had parameters of TR $4000 \mathrm{~ms}$, TE $90 \mathrm{~ms}$, and an echo-train length of 9 . Both the FLAIR and FSE sequences were acquired in the axial-oblique plane and had a FOV of $24 \mathrm{~cm}$, an imaging matrix of $256 \times 256$, 3 -mm-thick sections with a 3 -mm gap, and 24 sections that were a positioned so that their centers coincided with every other DWI section. A T1-weighted 3D spoiled gradient-recalled sequence was performed with parameters of TR $28 \mathrm{~ms}$, TE $6 \mathrm{~ms}$, flip angle $40^{\circ}, 256 \times$ 256 matrix, and 104 sections that were $1.5 \mathrm{~mm}$ thick.

\section{Image Analysis}

The data for each subject were deidentified and randomized. The data were in a DICOM format and were analyzed by using DICOM viewing software. ${ }^{32}$ An experienced rater (R.K.F.) blinded to subject identity and diagnosis evaluated the T1-weighted images, T2-weighted images, FLAIR images, DWI images, and ADC maps. ${ }^{8}$ Each sequence was evaluated separately to determine the presence or absence of signal intensity abnormalities in the brain. The judgment of signal intensity was made relative to uninvolved tissue and was graded as being normal or abnormal.

For each of the 5 imaging sequences, 61 brain regions were evaluated in the cerebrum, midbrain, pons, and cerebellum. The brain regions were as follows: the gray matter and subcortical white matter in each cerebral lobe (frontal, parietal, temporal, and occipital) and in the cingulate gyrus; the insula, hippocampus, and amygdala; the caudate nucleus, globus pallidus, putamen, thalamus, and hypothalamus; the corpus callosum, inferior longitudinal fasciculus, superior longitudinal fasciculus, and fronto-occipital fasciculus; the anterior, genu, and posterior limbs of the internal capsule; the anterior, middle, and posterior aspects of the corona radiata; the cerebellar hemispheres and the cerebellar peduncles; and the pons, midbrain, and corpus callosum. Structures that were in both hemispheres were considered to be separate brain regions. For example, the right frontal gray matter was considered to be a separate region from the left frontal gray matter.

\section{Statistical Analysis}

Radiologic severity was defined as the sum of abnormal ratings based on signal intensity changes across all of the regions and sequences. A contingency analysis with the $\chi^{2}$ test evaluated the sensitivity and specificity of the imaging sequences for each brain region, with the significance determined by the likelihood ratio method. The $\chi^{2}$ test is a standard statistical method to assess the distribution of a categoric response variable $\mathrm{Y}$ as conditioned by the values of a categoric factor $\mathrm{X}$. In this study, the $\chi^{2}$ test evaluates whether the frequency of the diagnostic groups (CJD versus control) is different due to the presence or absence of abnormal MR imaging signal intensity in a given structure.

\section{Results}

The final samples were well matched for age ( $61 \pm 8$ years for patients; $60 \pm 7$ years for controls) and did not differ significantly in sex distribution (45\% women among controls; $27 \%$ women among patients; $P=.24$ ). MR imaging was acquired early in the course of disease: the mean duration from onset of first symptom to examination was $8 \pm 15$ months, including 1 patient of unusually long duration (case 2007, see below). Without this patient, mean duration was $4 \pm 5$ months, with $50 \%$ of the patients seen within 3 months of onset.

The contingency analysis demonstrated that MR imaging is sensitive to fCJD caused by the E200K mutation (on-line Table). Twenty of the 61 brain regions demonstrated significant differences between CJD subjects and controls in one of these regions on at least one of the imagining sequences. These regions included the frontal lobe gray matter, frontal lobe white matter, parietal lobe gray matter, occipital lobe gray matter, occipital lobe white matter, cingulate gyrus gray matter, hippocampus, insula, caudate nucleus, putamen, globus pallidus, and thalamus (Fig 1). The brain regions involved in the highest percentage of fCJD cases in any single sequence were as follows: the caudate nuclei with FLAIR (12 subjects, 80\%), the caudate nuclei with DWI (11 subjects, $73 \%)$, the putamen with DWI (10 subjects, 67\%), the putamen with FLAIR (9 subjects, $60 \%$ ), the thalamus with DWI (6 subjects, $40 \%$ ), and the cingulate gyrus with DWI ( 6 subjects, $40 \%$ ). Imaging findings in cortical gray matter were best detected with DWI and were seen in the frontal gray matter (4 subjects, 27\%) and the occipital gray matter (3 subjects, 20\%). There were no signal intensity abnormalities in the cerebellum that differentiated fCJD patients and control subjects. fCJD subjects had some degree of atrophy, though cortical and ventricular volumes 

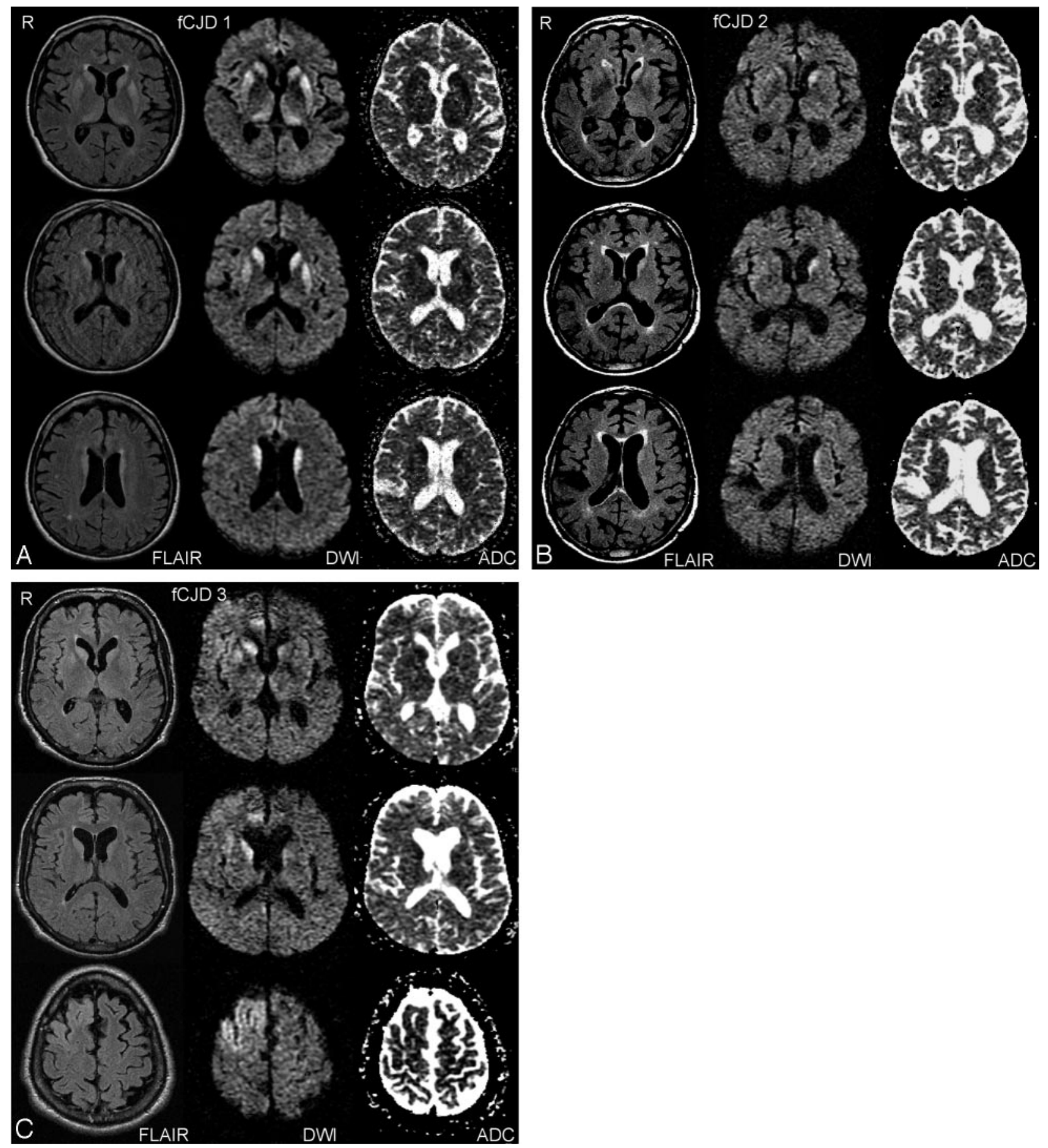

Fig 1. Representative imaging findings on FLAIR, DWI, and ADC sequences in 3 different subjects with familial CJD. In $A$ (fCJD 1), signal intensity abnormality involves the caudate nucleus, putamen, and thalamus, bilaterally. $B$ (fCJD 2) demonstrates abnormal signal intensity primarily in the left caudate nucleus. In $C$ (fCJD 3 ), there is involvement of both caudate nuclei, right greater than left, right cingulate gyrus, and right frontal lobe.

were not quantified in this study. Two of $15 \mathrm{fCJD}$ patients did not demonstrate signal intensity abnormalities on MR imaging in this blind analysis (except mild atrophy) and were considered to be false-negative by MR criteria (Fig 2, see Discussion). In 2 control subjects, the signal intensity changes seen in the caudate, putamen, and thalamus were focal areas of hyperintensity most consistent with small-vessel ischemic disease and lacunar infarcts.

The best discrimination between fCJD patients and control subjects was obtained by considering FLAIR in the right or left caudate nucleus or right or left putamen (on-line Table). Using this measure, sensitivity was $87 \%$ (13 of 15 subjects), and specificity was $91 \%$. The second best measure, FLAIR in either the right or left caudate nucleus, yielded a sensitivity of $80 \%$ and a specificity of $100 \%$. DWI also demonstrated good discrimination between the groups, reaching a sensitivity of $73 \%$ and a specificity of $100 \%$ in the combined caudate and putamen. T2-weighted and T1-weighted sequences demonstrated relatively low sensitivity (range of $0 \%-53 \%$ ). 


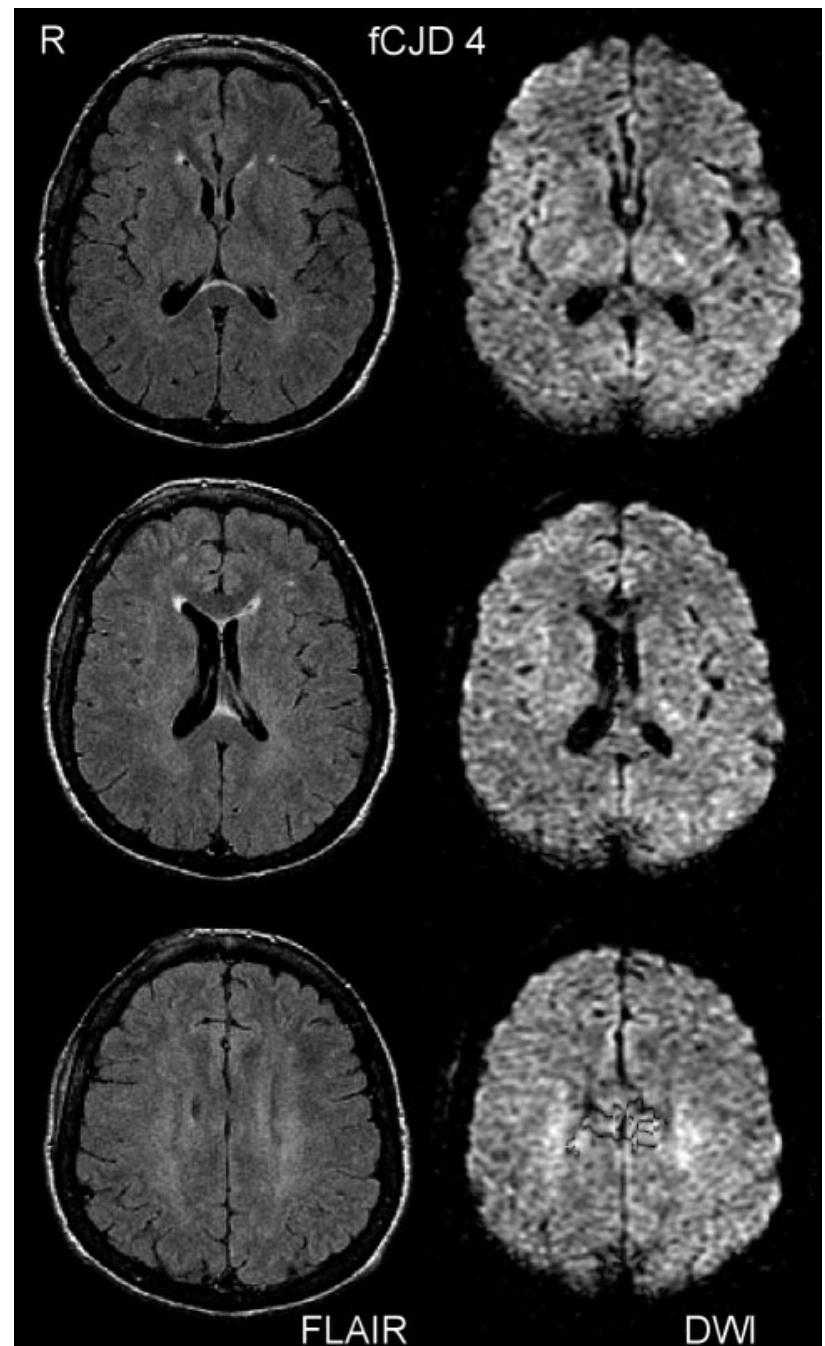

Fig 2. This subject (fCJD 4, case 2012) was rated as normal by FLAIR and DWI and was considered to be a false-negative by MR criteria (see Discussion). The ADC images (not shown) appeared normal.

\section{Discussion}

This study was the first to use blind ratings and healthy, agematched control subjects from the same families to determine the MR imaging findings in a relatively large number of subjects with fCJD caused by the E200K mutation. Using FLAIR and DWI, our study determined that up to $87 \%$ of fCJD subjects have abnormal MR studies. The brain regions most frequently involved (in descending order) were the caudate nucleus, putamen, thalamus, cingulate gyrus, and cerebral cortex. fCJD was optimally detected by FLAIR and DWI signal intensity hyperintensity in the caudate nucleus and putamen. FLAIR hyperintensity in the caudate or putamen resulted in a sensitivity of $87 \%$ and a specificity of $78 \%$. DWI signal intensity abnormality in the caudate yielded a sensitivity of $73 \%$ and a specificity of $100 \%$. Retrospective investigations (see below) suggest even better discrimination than these rigorous figures.

Previous imaging studies of fCJD consist mainly of case reports and encompass fCJD patients with a variety of point mutations, not just the type seen in E200K cases. ${ }^{15-19}$ A collaborative surveillance project of genetic prion cases indicated that approximately $50 \%$ of a small number (not specified) of patients with fCJD due to the E200K mutation had abnormal
MR images, but specific imaging results were not described. ${ }^{33}$ There are also larger imaging studies focused on SCJD that combined a few fCJD patients with sCJD patients into a single subject group but report the MR findings of the combined sample. ${ }^{8,20-22}$ The case reports on fCJD describe a spectrum of MR findings, with various degrees of basal ganglia, thalamic, and cortical involvement. Although the number of fCJD cases reported in the literature is small and limits the ability to rigorously compare results, the distributions of abnormal FLAIR and DWI signal intensity changes that we describe are consistent with the past case reports. Our results, along with the previous reports of fCJD, also suggest that the imaging findings in fCJD are in general similar to those seen in sCJD. ${ }^{5,15,17,19-22,33,34}$ These results are consistent with fCJD having clinical and neuropathologic features seen in the most common molecular subtype of sCJD, which is the MM1 subtype. $^{14}$

The basal ganglia signal intensity changes are important markers for CJD. This area of the brain is usually abnormal more than any other region, though the distribution of signal intensity changes can vary across cases and can differ within an individual over time..$^{8,11,19,35}$ That FLAIR and DWI are the best sequences to use in evaluation of fCJD supports previous studies of sCJD. ${ }^{21,22,36,37}$ In one study of sCJD patients with an average symptom duration of 12 months at the time of MR imaging, DWI and FLAIR were 91\% sensitive, 95\% specific, and $94 \%$ accurate in diagnosing sCJD. ${ }^{22}$ Without FLAIR or DWI sequences, sensitivity and specificity of MR imaging were $63 \%$ and $88 \%$, respectively. ${ }^{38}$ FLAIR and DWI sequences should be used in any imaging investigation of CJD.

MR signal intensity abnormality in the cortex was present in up to $24 \%$ of fCJD patients in our study but was not seen in the cerebellum. The percentage of cortical involvement is similar to the percentage reported by other studies of sCJD, which ranges from $24 \%$ to $45 \% .^{22,37}$ DWI and FLAIR sequences were best at detecting the cortical signal intensity changes corresponding with previous reports on the use of these 2 imaging sequences. ${ }^{21,22,36,37,39}$ Cortical signal intensity hyperintensity can change over time, however, and might not be detected unless careful serial imaging is performed. .,11 $^{8}$ Furthermore, when using cortical DWI and FLAIR hyperintensity for diagnostic purposes, it must be kept in mind that the insula and cingulate can normally have an increased signal intensity that is probably due to the cytoarchitecture of these regions. $22,35,40,41$

Thalamic hyperintensity is often associated with variant CJD (vCJD), but it is not unique to it. Signal intensity abnormality in the thalamus was seen in $40 \%$ of fCJD cases in our study and is also seen in sCJD. ${ }^{37,42,43}$ In vCJD, the thalamic signal intensity on FLAIR is generally believed to be brighter or of greater magnitude compared with the signal intensity of the caudate and putamen. ${ }^{44}$ In SCJD, the thalamic signal intensity is felt to be equal or lower in magnitude compared with the caudate and putamen. We did not directly compare the signal intensity of abnormal regions with other abnormal regions, but, in general, the thalamic signal intensity seen in the fCJD subjects in our study more closely resembled the SCJD pattern.

The pathologic basis of the abnormal signal intensity seen on MR is not completely known. It is believed to result from gliosis, spongiform degeneration, or accumulation of the ab- 
normal prion protein or from a combination of these factors. FLAIR hyperintensity was correlated with gliosis detected on pathologic evaluation. ${ }^{4,45}$ Another study reported that abnormal prion protein was associated with the increased signal intensity. ${ }^{46}$ With DWI, spongiform degeneration was confirmed histologically in patients with decreased water diffusion in the basal ganglia. ${ }^{16}$ Accumulation of the abnormal prion protein also corresponded with signal intensity hyperintensities seen on DWI. ${ }^{46}$ A case report of DWI correlation with histology found reduced ADC values in all of the regions with histologic alterations, but there was no correlation between ADC and spongiosis, neuronal loss, or gliosis. ${ }^{47} \mathrm{DWI}$ might be sensitive to functional changes that are not recognized on histology, as evidenced by a study reporting that abnormal DWI changes were lateralized to a hemisphere, though a subsequent autopsy revealed no asymmetric findings. ${ }^{12}$

ADC maps alone were not helpful in visually detecting abnormal signal intensity. ADC maps are best used in quantitative analyses of water diffusion rates in tissue. For example, a previous report using a region of interest analysis of diffusion changes in the basal ganglia demonstrated a statistically significant decrease in ADC values in the putamen in subjects with fCJD. ${ }^{27}$ ADC maps can also help confirm if the DWI signal intensity changes are due to a decreased rate of water diffusion or are a result of both a decreased rate of water diffusion and increased $\mathrm{T} 2$ signal intensity.

Our blind ratings failed to identify typical MR signal intensity abnormalities in 2 patients diagnosed with fCJD. Both subjects were relatively young. The first (case 2007) was a 53year-old woman with an unusually long history of 4 years and significant psychiatric symptoms, including brothers and a child being diagnosed as schizophrenic. The long clinical history and the overlying psychiatric symptoms made diagnosis difficult, though she was positive for the E200K mutation and had high CSF $\tau$ levels. On MR, the subject had atrophy and subtle signal intensity hyperintensity in the corona radiata bilaterally. Three years before this examination, a diagnosis of Alzheimer disease was considered. On retrospective evaluation, we quantified the changes of brain morphology in this patient between her initial MR imaging scan (analyzed in the current study) and another scan obtained 3 years later. The analysis used the high dimensional warping tool provided by SPM5 (Wellcome Department of Cognitive Neurology, London, United Kingdom, http://www.fil.ion.ucl.ac.uk/spm/) to warp the initial scan to match the subsequent scan as close as possible. The amount of regional contraction was extracted from the deformation field by taking the Jacobian determinant at each point. ${ }^{48}$ The results demonstrated that maximal atrophy occurred in the hippocampal formation, as is typical in Alzheimer disease. Although she was diagnosed as having fCJD for our prospective study, which resulted in a false-negative rating by MR, the negative MR findings in our results, the retrospective MR volume analyses, and the clinical course suggest that this patient may have been misdiagnosed as having fCJD, because she is still alive and no autopsy or biopsy data are available. Thus, the MR ratings in our study might be more appropriately considered to be true-negative, as her clinical and imaging data are not consistent with fCJD.

The second negative subject (case 2012, fCJD 4 in Fig 2) was a 54-year-old woman with a 6-month history that started with sleep disturbances and chronic fatigue. At the time of examination she was cognitively intact (MMS 27/30) but displayed central deafness and other severe neurologic symptoms, many of presumed cerebellar and brain stem origin. ${ }^{49}$ This subject only had mild atrophy on MR (Fig 2). However, on retrospective examination of DTI trace-weighted images (which we had started to acquire but did not use in the current study), a typical pattern of hyperintense caudate and pulvinar was seen bilaterally. This finding suggests that DTI might offer improved accuracy compared with DWI in the evaluation of CJD. ${ }^{50,51}$

Most studies of larger numbers of CJD subjects have a small percentage of cases that are negative on MR imaging. ${ }^{21,22,52}$ There are several possible explanations for the false-negative MR results. As noted above, imaging findings can evolve during the course of the disease, ${ }^{8,11}$ suggesting that serial imaging is important if the initial MR examination is negative. Patient motion can also obscure subtle findings, though DWI is usually robust. Furthermore, at least one of our false-negative subjects was cognitively intact with primarily cerebellar symptoms, and preliminary evidence suggests that the cerebellum might appear normal in nonquantitative MR imaging even in the presence of documented cerebellar symptoms. $^{51}$

There were several limitations to our study. A single rater was used, which could lead to bias, though our design used completely blinded ratings, and the sensitivity and specificity that we report are in line with previous studies, suggesting that bias was minimal. Our analysis included the first MR examination and did not have serial imaging to help determine how signal intensity abnormalities evolve. The fCJD subjects were not imaged at the same time in the course of their illness, which could account for variability in the imaging findings. Quantitative methods were not used to assess signal intensity changes, and histologic correlation was not available, though 14 of 15 patients were followed until death to confirm the diagnosis. The sample size was relatively small, making it difficult to extrapolate our findings; however, given the rarity of $\mathrm{fCJD}$, this is the largest imaging study to date of a homogenous sample of familial, or genetic, CJD cases.

\section{Conclusions}

Caudate and putamen signal intensity abnormality on FLAIR and DWI optimally differentiated fCJD patients from control subjects. MR imaging did not detect cerebellar abnormalities in fCJD subjects. The relatively high sensitivity and specificity that we report under strictly controlled and blinded conditions support the diagnostic use of MR imaging in fCJD.

\section{Acknowledgments}

We gratefully acknowledge contributions to this work by Janet Ben Mordechai, RN, Sheba Medical Center, and discussions with Dr Peter Kingsley, North Shore University Hospital.

\section{References}

1. Johnson RT, Gibbs CJ Jr. Creutzfeldt-Jakob disease and related transmissible spongiform encephalopathies. N Engl J Med 1998;339:1994-2004

2. Chapman J, Ben-Israel J, Goldhammer Y, et al. The risk of developing Creutzfeldt-Jakob disease in subjects with the PRNP gene codon 200 point mutation. Neurology 1994;44:1683-86

3. Bahn MM, Parchi P. Abnormal diffusion-weighted magnetic resonance images in Creutzfeldt-Jakob disease. Arch Neurol 1999;56:577-83 
4. Demaerel P, Baert AL, Vanopdenbosch L, et al. Diffusion-weighted magnetic resonance imaging in Creutzfeldt-Jakob disease. Lancet 1997;349:847-48

5. Finkenstaedt M, Szudra A, Zerr I, et al. MR imaging of Creutzfeldt-Jakob disease. Radiology 1996;199:793-98

6. Mao-Draayer Y, Braff SP, Nagle KJ, et al. Emerging patterns of diffusionweighted MR imaging in Creutzfeldt-Jakob disease: case report and review of the literature. AJNR Am J Neuroradiol 2002;23:550-56

7. Matoba $M$, Tonami $H$, Miyaji $H$, et al. Creutzfeldt-Jakob disease: serial changes on diffusion-weighted MRI. J Comput Assist Tomogr 2001;25:274-77

8. Murata T, Shiga Y, Higano S, et al. Conspicuity and evolution of lesions in Creutzfeldt-Jakob disease at diffusion-weighted imaging. AJNR Am J Neuroradiol 2002;23:1164-72

9. Na DL, Suh CK, Choi SH, et al. Diffusion-weighted magnetic resonance imaging in probable Creutzfeldt-Jakob disease: a clinical-anatomic correlation. Arch Neurol 1999;56:951-57

10. Samman I, Schulz-Schaeffer WJ, Wohrle JC, et al. Clinical range and MRI in Creutzfeldt-Jakob disease with heterozygosity at codon 129 and prion protein type 2. J Neurol Neurosurg Psychiatry 1999;67:678-81

11. Ukisu R, Kushihashi T, Kitanosono T, et al. Serial diffusion-weighted MRI of Creutzfeldt-Jakob disease. AJR Am J Roentgenol 2005;184:560-66

12. Yee AS, Simon JH, Anderson CA, et al. Diffusion-weighted MRI of right-hemisphere dysfunction in Creutzfeldt-Jakob disease. Neurology 1999;52:1514-15

13. Yoon SS, Chan S, Chin S, et al. MRI of Creutzfeldt-Jakob disease: asymmetric high signal intensity of the basal ganglia. Neurology 1995;45:1932-33

14. Gambetti P, Kong Q, Zou W, et al. Sporadic and familial CJD: classification and characterisation. Br Med Bull 2003;66:213-39

15. Ishida S, Sugino M, Koizumi N, et al. Serial MRI in early Creutzfeldt-Jacob disease with a point mutation of prion protein at codon 180. Neuroradiology 1995;37:531-34

16. Mittal S, Farmer P, Kalina P, et al. Correlation of diffusion-weighted magnetic resonance imaging with neuropathology in Creutzfeldt-Jakob disease. Arch Neurol 2002;59:128-34

17. Nitrini R, Mendonca RA, Huang N, et al. Diffusion-weighted MRI in two cases of familial Creutzfeldt-Jakob disease. J Neurol Sci 2001;184:163-67

18. Satoh A, Goto H, Satoh H, et al. A case of Creutzfeldt-Jakob disease with a point mutation at codon 232: correlation of MRI and neurologic findings. Neurology 1997;49:1469-70

19. Tsuboi Y, Baba Y, Doh-Ura K, et al. Diffusion-weighted MRI in familial Creutzfeldt-Jakob disease with the codon 200 mutation in the prion protein gene. J Neurol Sci 2005;232:45-49

20. Lin YR, Young GS, Chen NK, et al. Creutzfeldt-Jakob disease involvement of rolandic cortex: a quantitative apparent diffusion coefficient evaluation. AJNR Am J Neuroradiol 2006;27:1755-59

21. Shiga Y, Miyazawa K, Sato S, et al. Diffusion-weighted MRI abnormalities as an early diagnostic marker for Creutzfeldt-Jakob disease. Neurology 2004;63: 443-49

22. Young GS, Geschwind MD, Fischbein NJ, et al. Diffusion-weighted and fluidattenuated inversion recovery imaging in Creutzfeldt-Jakob disease: high sensitivity and specificity for diagnosis. AJNR Am J Neuroradiol 2005;26: $1551-62$

23. Bergui M, Bradac GB, Rossi G, et al. Extensive cortical damage in a case of Creutzfeldt-Jacob disease: clinicoradiological correlations. Neuroradiology 2003;45:304-07

24. Goldfarb LG, Korczyn AD, Brown P, et al. Mutation in codon 200 of scrapie amyloid precursor gene linked to Creutzfeldt-Jakob disease in Sephardic Jews of Libyan and non-Libyan origin. Lancet 1990;336:637-38

25. Hsiao K, Meiner Z, Kahana E, et al. Mutation of the prion protein in Libyan Jews with Creutzfeldt-Jakob disease. $N$ Engl J Med 1991;324:1091-97

26. Kahana E, Alter M, Braham J, et al. Creutzfeldt-Jakob disease: focus among Libyan Jews in Israel. Science 1974;183:90-91

27. Fulbright RK, Kingsley $\mathrm{PB}$, Guo $\mathrm{X}$, et al. The imaging appearance of Creutzfeldt-Jakob disease caused by the E200K mutation. Magn Reson Imaging 2006;24:1121-29

28. Satoh K, Shirabe S, Tsujino A, et al. Total tau protein in cerebrospinal fluid and diffusion-weighted MRI as an early diagnostic marker for Creutzfeldt-Jakob disease. Dement Geriatr Cogn Disord 2007;24:207-12
29. Goldgaber D, Goldfarb LG, Brown P, et al. Mutations in familial CreutzfeldtJakob disease and Gerstmann-Straussler-Scheinker's syndrome. Exp Neurol 1989;106:204-06

30. Folstein MF, Folstein SE, McHugh PR. "Mini-mental state." A practical method for grading the cognitive state of patients for the clinician. J Psychiatr Res 1975;12:189-98

31. Chapman J, Cohen OS, Ephraty L, et al. The CJD rating scale: a new tool for evaluation of neurologic symptomatology in early CJD patients [abstract]. Presented at the 59th Annual Meeting of the American Academy of Neurology, April 28-May 5, 2007; Boston, Mass;A140

32. Rosset A, Spadola L, Pysher L, et al. Informatics in radiology (infoRAD): navigating the fifth dimension: innovative interface for multidimensional multimodality image navigation. Radiographics 2006;26:299-308

33. Kovacs GG, Puopolo M, Ladogana A, et al. Genetic prion disease: the EUROCJD experience. Hum Genet 2005; 118:166-74

34. Tschampa HJ, Kallenberg K, Urbach H, et al. MRI in the diagnosis of sporadic Creutzfeldt-Jakob disease: a study on inter-observer agreement. Brain 2005; 128:2026-33

35. Tschampa HJ, Zerr I, Urbach H. Radiological assessment of Creutzfeldt-Jakob disease. Eur Radiol 2007;17:1200-11

36. Zeidler M, Collie DA, Macleod MA, et al. FLAIR MRI in sporadic CreutzfeldtJakob disease. Neurology 2001;56:282

37. Kallenberg K, Schulz-Schaeffer WJ, Jastrow U, et al. Creutzfeldt-Jakob disease: comparative analysis of MR imaging sequences. AJNR Am J Neuroradiol 2006;27:1459-62

38. Meissner B, Kortner K, Bartl M, et al. Sporadic Creutzfeldt-Jakob disease: mag netic resonance imaging and clinical findings. Neurology 2004;63:450-56

39. Tschampa HJ, Kallenberg K, Kretzschmar HA, et al. Pattern of cortical changes in sporadic Creutzfeldt-Jakob disease. AJNR Am J Neuroradiol 2007;28: $1114-18$

40. Hirai T, Korogi Y, Yoshizumi K, et al. Limbic lobe of the human brain: evaluation with turbo fluid-attenuated inversion-recovery MR imaging. Radiology 2000;215:470-75

41. Asao C, Hirai T, Yoshimatsu S, et al. Human cerebral cortices: signal variation on diffusion-weighted MR imaging. Neuroradiology 2008;50:205-11

42. Martindale J, Geschwind MD, De Armond S, et al. Sporadic Creutzfeldt-Jakob disease mimicking variant Creutzfeldt-Jakob disease. Arch Neurol 2003;60:767-70

43. Tschampa HJ, Murtz P, Flacke S, et al. Thalamic involvement in sporadic Creutzfeldt-Jakob disease: a diffusion-weighted MR imaging study. AJNR Am J Neuroradiol 2003;24:908-15

44. Haik S, Brandel JP, Oppenheim C, et al. Sporadic CJD clinically mimicking variant CJD with bilateral increased signal in the pulvinar. Neurology 2002;58:148-49

45. Urbach H, Klisch J, Wolf HK, et al. MRI in sporadic Creutzfeldt-Jakob disease correlation with clinical and neuropathological data. Neuroradiology 1998;40:65-70

46. Haik S, Dormont D, Faucheux BA, et al. Prion protein deposits match magnetic resonance imaging signal abnormalities in Creutzfeldt-Jakob disease. Ann Neurol 2002;51:797-99

47. Russmann H, Vingerhoets F, Miklossy J, et al. Sporadic Creutzfeldt-Jakob disease. J Neurology 2005;252:338-42

48. Freeborough PA, Fox NC. Modeling brain deformations in Alzheimer disease by fluid registration of serial 3D MR images. J Comput Assist Tomogr 1998;22:838-43

49. Rene R, Campdelacreu J, Ferrer I, et al. Familial Creutzfeldt-Jakob disease with E200K mutation presenting with neurosensorial hypoacusis. J Neurol Neurosurg Psychiatry 2007;78:103-04

50. Hoffman CC, Fulbright RK, Konen E, et al. Early detection of familial JakobCreutzfeldt disease: comparison of T-2, FLAIR and DTI techniques. Presented at the Radiological Society of North America, December 1, 2006; Chicago, Ill.

51. Cohen OS, Hoffman C, Chapman J, et al. Correlation between DTI and neurological symptoms in patients with familial Creutzfeldt-Jakob disease [abstract]. Presented at the 59th Annual Meeting of the American Academy of Neurology, 2007; Boston, Mass;A140

52. Schroter A, Zerr I, Henkel K, et al. Magnetic resonance imaging in the clinical diagnosis of Creutzfeldt-Jakob disease. Arch Neurol 2000;57:1751-57 